\title{
Nonspatial attributes of stimuli can influence spatial limitations of attentional control
}

\author{
Cathleen M. Moore, Lyndey K. Lanagan-Leitzel, Peggy Chen, and Rose Halterman \\ Pennsylvania State University, University Park, Pennsylvania \\ AND \\ ELisAbeth M. Fine \\ Schepens Eye Research Institute and Harvard Medical School, Cambridge, Massachusetts
}

\begin{abstract}
A wide variety of psychophysical and neurophysiological research suggests that when stimuli are very close together, they cannot be attended separately. As a consequence, they cannot be represented as individual items with specific feature information associated with them. Here we report evidence that the spatial control of attention can be modulated by nonspatial features of the stimuli (such as color and luminance). Observers shifted attention from item to item within highly dense arrays of stimuli. Performance was extremely poor when all of the items in the array were an identical gray. In contrast, performance improved when items differed in color. This finding indicates that nonspatial features, such as color, can facilitate spatial selection and suggests moreover that features can be reliably associated with particular items even when the items are densely clustered.
\end{abstract}

Work from a variety of approaches suggests that the selection and representation of visual information is subject to fairly severe spatial limitations. These limitations are believed to reflect the structure and function of basic visual information-processing mechanisms, including the formation and individuation of coherent object representations (see, e.g., Cavanagh, 2001; C. W. Eriksen \& Hoffman, 1973; He, Cavanagh, \& Intriligator, 1996, 1997; Intriligator \& Cavanagh, 2001; Pelli, Palomares, \& Majaj, 2004). Here we report evidence that the spatial control of attention can be modulated by nonspatial features (such as color and luminance) of the stimuli.

Intriligator and Cavanagh (2001) used the term "attentional resolution" to define the smallest possible interitem distance that still allows observers to successfully shift attention from one item to another. They measured attentional resolution using a task that we will refer to as the attentional walk. In one condition, displays consisted of circular arrays of identical disks that varied in density and eccentricity. While observers fixated a central point, one of the disks briefly changed color, indicating that attention should be shifted to that disk. Following this cue, a series of verbal commands instructed the observers to move attention one disk to the left or to the right of the disk they were currently attending (e.g., "left, left, right, left," etc.). At the end of a five- to seven-step series of commands, another disk was probed by having it change colors. The observers' task was to indicate whether or not the probed disk was the end point of the attentional walk. The logic was that if the observers were able to reliably shift their attention within the array, then they would be able to follow the walk and indicate correctly where it ended. If, however, the density of the array was beyond the resolution of attentional control, then the observers would be unable to move through the array successfully and therefore unable to indicate where the walk ended. Notice that performance in the attentional walk task reflected not only the observers' ability to select an individual item, but also their ability to use that information to successfully shift attention from one item to another within arrays of items; these abilities may be separable processing limitations (Moore, Lanagan-Leitzel, \& Fine, 2007).

Using the attentional walk task, Intriligator and Cavanagh (2001) found that observers could easily perceive individual items within a broad range of display densities, yet they could not reliably report the end point of an attentional walk. In addition, they found that attentional resolution, as measured using the attentional walk, tended to be coarser in the upper visual field than in the lower visual field (see also He et al., 1996), and that it tended to be coarser with increasing eccentricity. These findings are consistent with the idea that spatial attention is spatially limited.

In conceptualizing the limitations of attentional resolution, Intriligator and Cavanagh (2001) suggested that when two items fall within a critically small region, those items cannot be individuated. They suggested that the limitation might reflect representational pointers mediated by parietal cortex neurons, which possess relatively large receptive fields, and that therefore "visual areas further along the stream of processing would not be able to de-

C. M. Moore, cmm15@psu.edu 
termine which properties went with which item within the region ... treat [ing] all properties from the selected region as belonging to one entity" (p. 211).

Pelli et al. (2004) introduced the construct of an "integration field," which shares some of the characteristics captured by the construct of attentional resolution. They proposed an account of visual crowding (i.e., the reduced ability to identify target stimuli when they are flanked by other, similar stimuli) that is based on a two-stage process of object identification. In the first stage, the features of objects are extracted from the visual scene. In the second stage, these features must be integrated into and associated with the appropriate object representations. Pelli et al. proposed that visual crowding is caused by a failure during the integration stage. Specifically, when more than one object is processed within a single critical region - the integration field - the features extracted from the separate objects cannot be correctly associated with their respective object representations, thus preventing correct identification of the objects. On the basis of the extensive literature on visual crowding and further studies presented in their article, Pelli et al. estimated the extent of integration fields to be about $0.5 \times$ the eccentricity at which the target is presented, a range they referred to as "Bouma's bound," since Bouma (1970) originally identified it as a critical range.

In addition to attentional resolution and integration fields, numerous other models have used spatial features to characterize the selection of visual information (see Cave \& Bichot, 1999, and Fernandez-Duque \& Johnson, 1999, for reviews) including a spotlight (see, e.g., Posner, 1980; Posner, Snyder, \& Davidson, 1980), a zoom lens (see, e.g., C. W. Eriksen \& St. James, 1986; C. W. Eriksen $\&$ Yeh, 1985), and various types of spatial filters (see, e.g., LaBerge \& Brown, 1989), including ones with central excitatory regions surrounded by adjacent inhibitory regions (see, e.g., Bahcall \& Kowler, 1999; Cutzu \& Tsotsos, 2003; Mounts, 2000; Steinman, Steinman, $\&$ Lehmkuhle, 1995). All of these models stipulate an extent of space in which information is selectively processed and within which further selectivity is impossible. A spotlight of attention, for example, is assumed to have a minimum size that can be measured by assessing the degree to which a stimulus can be processed without interference from nearby items (see, e.g., C. W. Eriksen \& Hoffman, 1973). Estimates of the size and shape of this spatial extent vary across different conditions and tasks, but the estimates are broadly in keeping with the notion that once the spatial separations among stimuli drop below a minimum distance, the stimuli can no longer be individually represented and processed.

Although attentional resolution, integration fields, and the various other models of spatial attention that have been put forward differ in important ways, most of them share a common implication. Specifically, most predict that adding differentiating feature information to stimuli (e.g., making the stimuli different colors) will facilitate the individuation of stimuli only if they are separated by more than the critical distance. For integration fields, for example, stimuli that are separated by distances smaller than the boundaries of the integration field should be no easier to identify if their features are distinct (e.g., different colors) than if their features are identical. According to the model, if two stimuli fall within a single integration field, then feature information cannot be reliably associated with any one item. If a feature cannot be reliably associated with any one item, then isolating any specific item from other items cannot help. Similarly, according to the attentional resolution model, giving stimuli within a highly dense array of items distinct features instead of identical ones would provide no advantage in the attentional walk task because items within highly dense arrays are not represented as individual items possessing specific features but as inaccessible components of a texture (Cavanagh, 2001; He, Cavanagh, \& Intriligator, 1997; Intriligator \& Cavanagh, 2001; Parkes, Lund, Angelucci, Solomon, \& Morgan, 2001). The various models that ascribe a limited extent of space to selection have similar implications.

In the present study, we investigated this prediction using an attentional walk task (Intriligator \& Cavanagh, 2001). We chose the attentional walk task because it was originally designed to measure the extent to which items can be individuated rather than identified. If items must be individuated in order for their features to be reliably associated with them, and if items cannot be individuated in highly dense arrays, then adding feature information to items in highly dense arrays should do little to facilitate performance in the attentional walk task. By way of preview, we found that performance in the attentional walk task was facilitated by adding distinguishing color information to the items.

\section{EXPERIMENT 1}

Observers were tested on a variation of the attentional walk task using arrays of varying density that comprised either differently colored disks (heterogeneous) or identically colored disks (homogeneous). ${ }^{1}$ If a nonspatial feature like color can be used to facilitate the individuation of items, then the effect of increasing density on performance in the attentional walk task should be less severe for the heterogeneous arrays than for the homogeneous arrays. In contrast, if no advantage is afforded by the addition of differentiating nonspatial feature information, then the effect of density on performance in the attentional walk task should be uninfluenced by heterogeneity.

\section{Method}

Observers. Sixteen individuals from the Pennsylvania State University community (mainly graduate students) served as observers. Three were authors, while the rest were naive to the purpose of the experiment. All reported normal or corrected-to-normal visual acuity and color vision.

Equipment. The experiment was controlled by an IBMcompatible PC using MATLAB software (version 6.5 release 13) with the Psychophysics Toolbox (Win Version 2.54) extensions (Brainard, 1997; Pelli, 1997). Stimuli were presented on a 17in. color monitor with a resolution of $1,024 \times 768$ and a refresh 
rate of $60 \mathrm{~Hz}$. Fixation was monitored for a subset of the observers $(n=5)$ using an Arrington Research video-based monocular eyetracker running Viewpoint software (Version PC60). Viewing distance was fixed for all subjects with an Arrington Quickclamp chin-and-headrest at $52 \mathrm{~cm}$

Stimuli. Stimuli were circular arrays of 12,18 , or 24 gray or colored disks (radius $=0.49^{\circ}$ ) presented at $7.5^{\circ}$ (to the center of a disk) eccentricity. The array was centered around a $0.33^{\circ}$ square fixation marker, which was presented at the center of the monitor. Arrays were presented on a dark field, with luminance of $3.24 \mathrm{~cd} / \mathrm{m}^{2}$, as measured with a Minolta LS100 light meter. Gray disks were $7.26 \mathrm{~cd} / \mathrm{m}^{2}$ (RGB [70 7070$\left.]\right)$. Colored disks were red $\left(6.7 \mathrm{~cd} / \mathrm{m}^{2}\right.$;

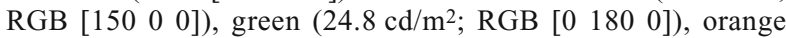
(24.19 cd/m²; RGB [241 127 0]), blue (7.97 cd/m²; RGB [0 0250$]$ ), and purple (12.48 cd/ $/ \mathrm{m}^{2}$; RGB [200 0 200]). The cue marking the beginning point of an attentional walk was a white $\left(132.84 \mathrm{~cd} / \mathrm{m}^{2}\right)$ annulus $\left(0.27^{\circ}\right.$ inner radius, $0.49^{\circ}$ outer radius) centered on top of one of the disks. The color of each disk for all colored arrays was constrained in such a way that no two adjacent disks were the same color, but otherwise colors were chosen randomly. The response arrays were always colored. In addition to the constraint that no two adjacent disks be the same color, an additional constraint required that the color of the target (the final disk in the attentional walk) be different from the colors of the two disks on either side of it. High $(800 \mathrm{~Hz})$ tones indicated each step in the walk, and a low $(500 \mathrm{~Hz})$ tone indicated the end of the walk.

Procedure and Task. Observers each completed two sessions. The first began with an oral description of the task from the experimenter along with a set of written instructions presented on the computer screen. Instructions emphasized that observers should remain fixated on the central fixation marker throughout the trial. After reading the instructions, participants completed at least 24 practice trials. For those observers whose fixation was monitored, eye position was calibrated at this point, and more practice trials were provided until the observer was comfortable with the eyetracker and was able to successfully hold fixation without blinking or otherwise moving throughout the trial. Six blocks of 24 trials each were then completed by all observers. The second session proceeded in the same way except that the instructions were merely briefly reviewed and only 12 practice trials were given.

Trial events are illustrated in Figure 1. Each trial began with the presentation of the central fixation square (the cue). The fixation display lasted for $1 \mathrm{sec}$ if fixation was not monitored or until the space bar was pressed if fixation was monitored. The array of disks was then added to the display, and $1 \mathrm{sec}$ later, the cue appeared for $1 \mathrm{sec}$. A series of 1, 5, 7, or 9 tones, all high except the last one (which was always low), was then played. When there was only one tone, it was always low. Each tone had a duration of $200 \mathrm{msec}$ and was followed by a $750-\mathrm{msec}$ silent interval. The array of disks changed colors $750 \mathrm{msec}$ after the final (low) tone to reveal the response display. In the homogeneous condition, this change was from an array of all gray disks to one of differently colored disks. In the heterogeneous condition, change was from one array of differently colored disks to a new array of differently colored disks.

Observers were asked to move their attention to the cued disk and to shift attention, but not fixation, one step clockwise (or counterclockwise) in response to each tone. Most trials (multiple-step trials) included a series of 5, 7, or 9 tones, starting with high tones and ending with a low tone. The low tone indicated the final step of the walk and that the response display was about to appear. On the remaining trials ( 0 -step trials), only a low tone occurred, and observers were to report the color of the disk at the cued location once the response display appeared.

Design. A 2 (display type: heterogeneous, homogeneous) $\times 3$ (density: $12,18,24$ disks) $\times 2$ (number of steps: zero, multiple) within-subjects design was used. Walk lengths were $0,5,7$, or 9 steps. For purposes of analysis, data from the 5-, 7-, and 9-step trials were collapsed into a single multiple-step condition. Half of the observers shifted attention in the clockwise direction and the other half shifted attention in the counterclockwise direction. Display type was manipulated across the two experimental sessions. All other variables were mixed within blocks of trials and were chosen randomly on each trial. Each subject participated in two six-block sessions. Because conditions were chosen randomly, the specific number
Homogeneous

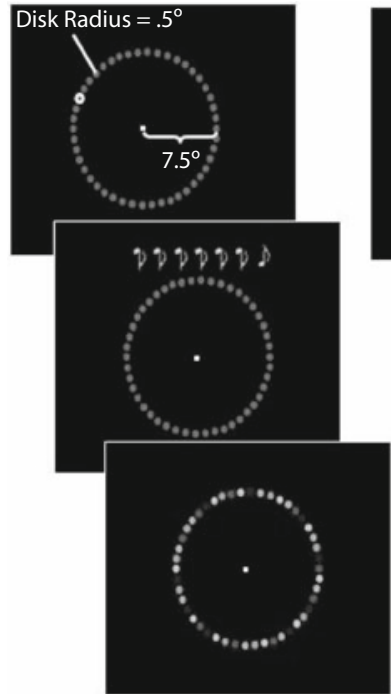

Heterogeneous

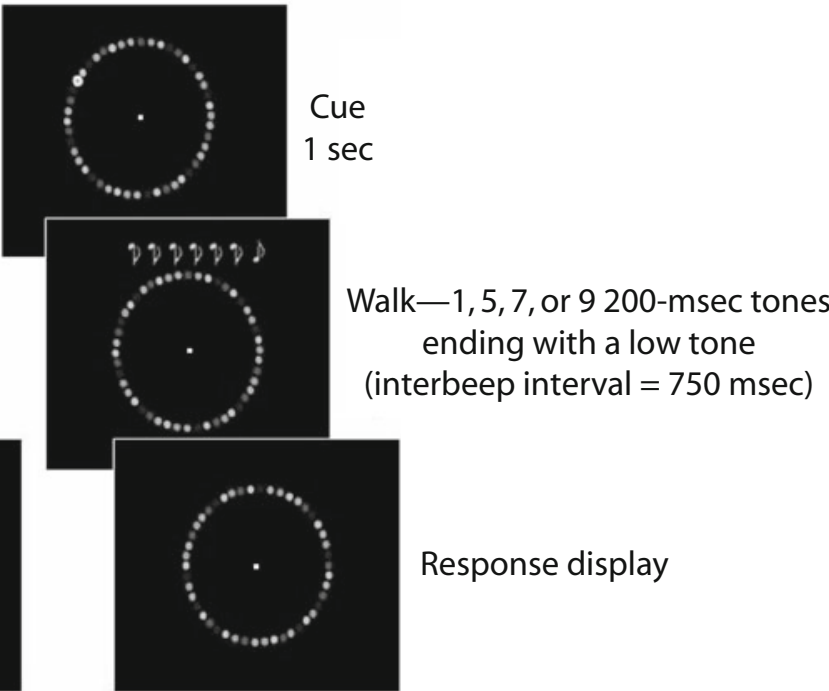

Figure 1. Trial events for the homogeneous (left) and heterogeneous (right) conditions. Each trial began with the presentation of the cue. The fixation display lasted for $1 \mathrm{sec}$. The array of disks was then added to the display, and 1 sec later, the cue appeared for 1 sec. A series of 1, 5, 7, or 9 tones, all high except the last one (which was always low), was then played. Each tone had a duration of $200 \mathrm{msec}$ and was followed by a $\mathbf{7 5 0}$-msec silent interval. The array of disks changed colors $750 \mathrm{msec}$ after the final (low) tone to reveal the response display. 
of observations varied. The mean number of observations per observer was 45 for the 0 -step conditions and 108 for the multiple-step conditions.

\section{Results and Discussion}

Data from two of the observers were excluded from the analysis because of near-chance performance in the 0 -step conditions. Figure 2 shows the mean percent correct across observers as a function of density. Circles represent the 0 -step conditions, and squares represent the multiple-step conditions, with filled circles and squares representing the homogeneous displays and open ones representing the heterogeneous displays. Observer means were submitted to a 2 (display type) $\times 3$ (density) $\times 2$ (number of steps) ANOVA. Alpha was set at 0.05 for these and all subsequent analyses. All sources of variance were reliable, including the three-way interaction $[F(2,26)=3.80, p<.05]$. Referring to Figure 2, one can see the nature of the threeway interaction, which was confirmed by two separate 2 (display type) $\times 3$ (density) ANOVAs conducted for the 0 - and multiple-step conditions. For the 0 -step condition, although the main effect of density was reliable $[F(1,13)=$ $18.08, p<.001]$, neither the main effect of display type $[F(1,13)=1.78$, n.s. $]$ nor the interaction between display type and density $[F(2,26)=0.21$, n.s.] was significant. In contrast, for the multiple-step condition, the main effects of both display type $[F(1,13)=65.76, p<.001]$ and density $[F(1,13)=13.95, p<.01]$ were reliable. Moreover, and most important for the present question, the interaction between display type and density was reliable $[F(2,26)=$ $9.22, p<.01]$, confirming that performance on the heterogeneous displays decreased less sharply as a function of density than did performance on the homogeneous displays in the multiple-step conditions.

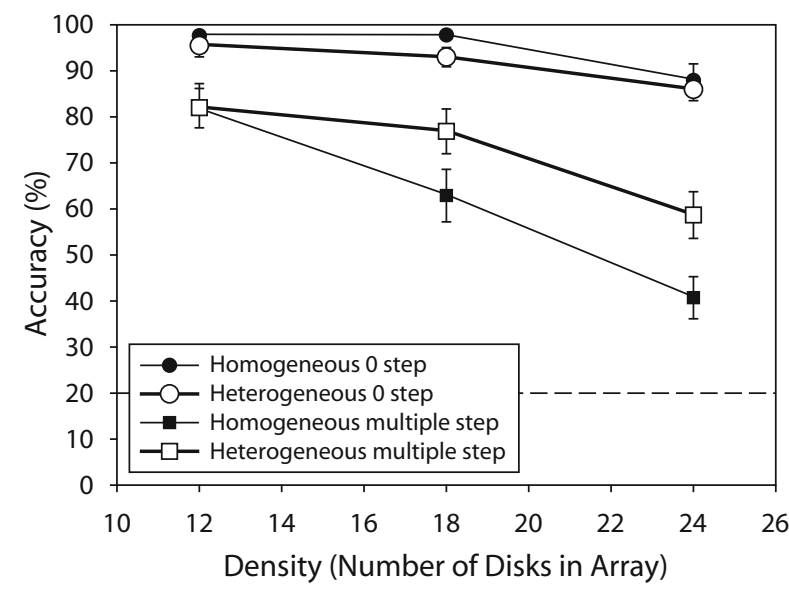

Figure 2. Mean percent correct shown as a function of the number of items in the array (density) from Experiment 1. Circles represent the 0 -step conditions and squares represent the multiple-step conditions, with filled circles and squares representing the homogeneous displays and open ones representing the heterogeneous displays. Error bars show the standard errors of the means across observers.
The same set of 2 (display type) $\times 3$ (density) analyses were conducted separately for the set of 5 observers whose fixation was monitored throughout the experiment. There were no differences in the data patterns between these observers and the group including all observers.

Figure 3 shows the probability of accurately reporting a given disk's color as a function of the position of that disk relative to the target for the 0-step (Figure 3A) and multiple-step (Figure 3B) conditions separately. Because disks were uniquely colored within a 5-disk span (target \pm 2 disks), data could be summarized in terms of which disk was reported. A caveat regarding this analysis is that it is possible that a nontarget color response was either a guess or was based on a disk outside of the target \pm 2 disk range. We assume that these cases were rare and that they were distributed randomly across the range of disks surrounding the target. There were very few errors in the 0 -step condition. Where errors occurred in the multiplestep condition, they were mainly reports of the color of a disk one disk away from the target.

In summary, observers' ability to shift attention within dense arrays was influenced by the heterogeneity of the items within the array. Consistent with previous findings from the attentional walk task used by Intriligator and Cavanagh (2001), observers' ability to move attention among items decreased as density increased. However, this decrease in performance due to increased density was less for heterogeneous displays than for homogeneous displays. This suggests that the addition of distinguishing nonspatial features to items in an array facilitates the individuation of items and the ability to shift attention among them.

\section{EXPERIMENT 2}

To generalize our findings, a second experiment with fewer observers but a broader range of densities was conducted. In addition to the increase in densities, display type was randomized within blocks of trials. The increased range of densities allowed us to track performance across a broader range and thereby confirm that the pattern of results observed in Experiment 1 was not unique to the particular range of densities used there. Randomizing display type within blocks of trials reduced concerns that differences in performance as a function of display type resulted from strategy choices induced by blocking in Experiment 1.

\section{Method}

Except where indicated, the method was identical to that in Experiment 1.

Observers. Three observers, all authors who had participated in Experiment 1, participated in Experiment 2.

Design and Procedure. A 2 (display type: heterogeneous, homogeneous) $\times 5$ (density: $7,14,21,28,35) \times 2$ (number of steps: zero, multiple) within-subjects design was used. Walk lengths were 0,7 , or 9 steps. Data from 7- and 9-step trials were collapsed into one multiple-step condition for analysis. All variables were manipulated within blocks of trials. Each trial type occurred once in a pseudorandom order within each 30-trial block. Participants completed four separate six-block sessions, each starting with a 15 -trial practice block. This resulted in 24 observations in the 0 -step condition and 

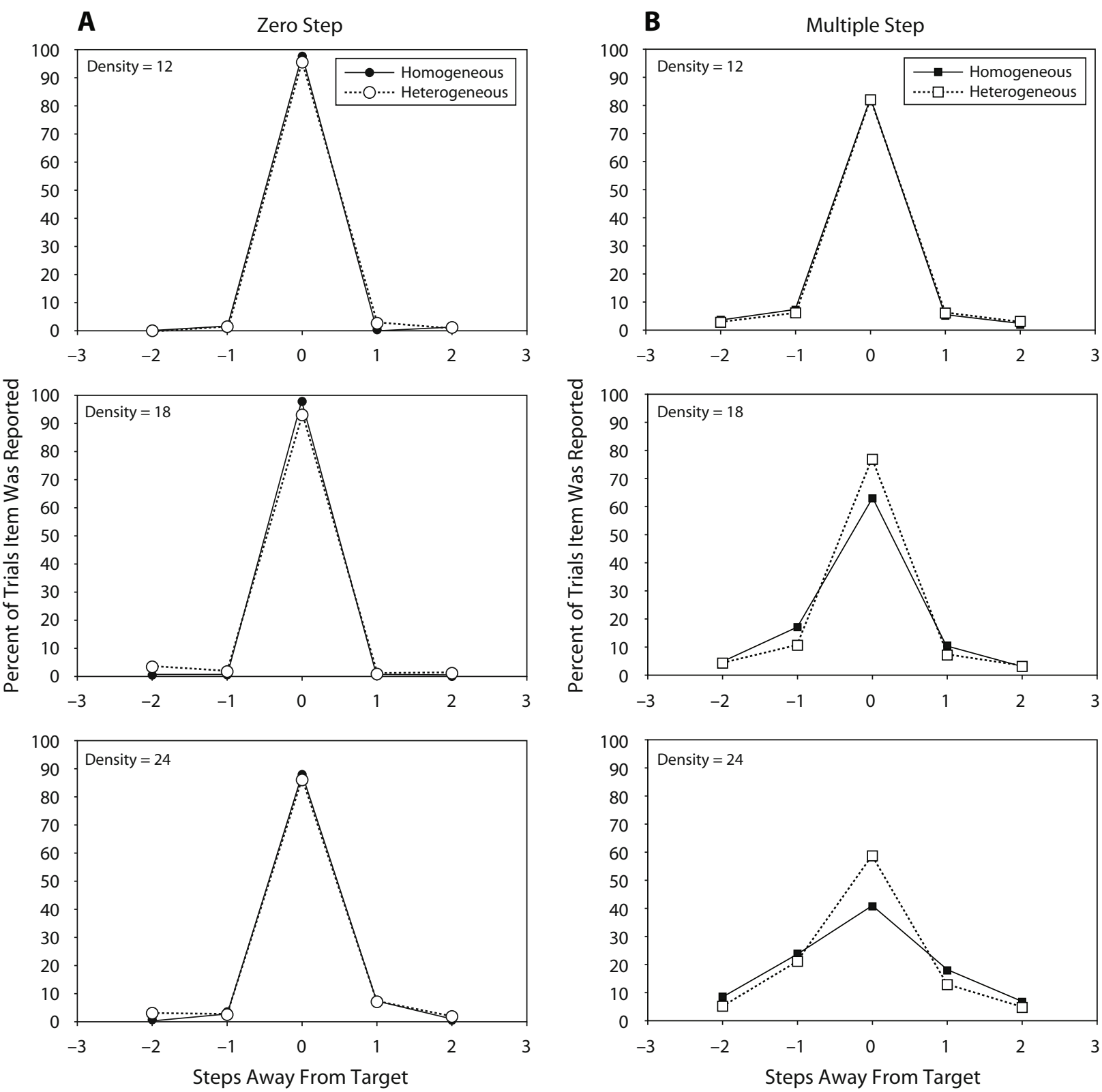

Figure 3. Probability of reporting the color of a given disk as a function of that disk's distance from the target disk for Experiment 1. Filled symbols represent homogeneous displays and open symbols represent heterogeneous displays. (A) Data for three different density levels in the 0-step conditions. (B) Data for three different density levels in the multiple-step conditions.

48 observations in each of the 7- and 9-step conditions, for each observer. Fixation was monitored for two of the observers.

\section{Results and Discussion}

Figure 4 shows the mean percent correct across observers as a function of density for the heterogeneous (open symbols) and homogeneous (filled symbols) conditions separately. The patterns are identical to those of Experiment 1 , although performance has been tracked through a broader range. In particular, the observers' ability to shift attention within arrays of items decreased with increasing density, but this decrease was less pronounced for heterogeneous arrays than for homogeneous arrays. Separate 2 (display type) $\times 5$ (density) ANOVAs were conducted for the 0- and multiple-step conditions. As in Experiment 1, only the main effect of density was reliable in the 0 -step condition $[F(1,13)=10.15, p<.01]$. Neither the main effect of display type $[F(1,2)=0.26$, n.s.] nor the display type $\times$ density interaction was reliable $[F(4,8)=0.24$, n.s.]. For the multiple-step condition, the main effect of density was reliable $[F(4,8)=30.00, p<.001]$, and although the main effect of display type was not reliable with only 3 observers $[F(1,2)=8.98, p<.10]$, the display type $\times$ density interaction was $[F(4,8)=4.43, p<$ $.01]$. This reliable interaction, like that in Experiment 1, confirms that performance in the attentional walk task de- 


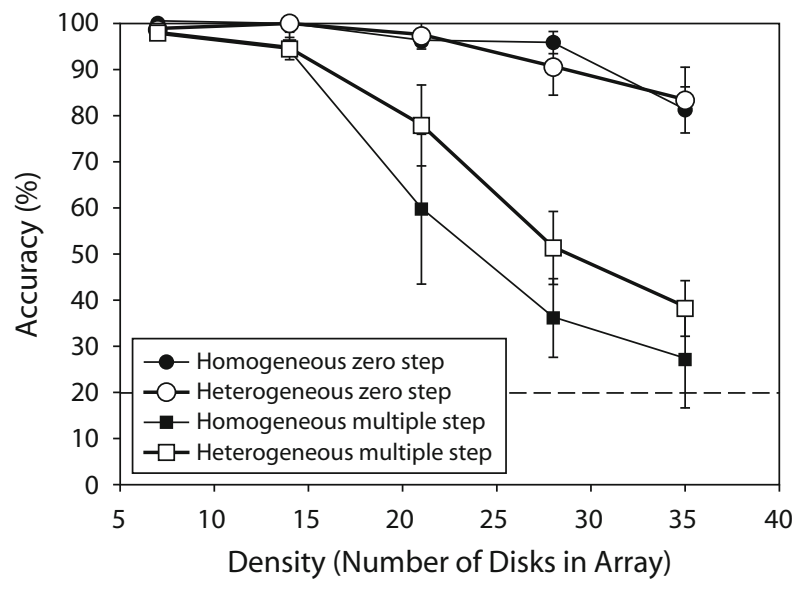

Figure 4. Mean percent correct shown as a function of the number of items in the array (density) from Experiment 2. Circles represent the 0 -step conditions and squares represent the multiple-step conditions, with filled circles and squares representing the homogeneous displays and open ones representing the heterogeneous displays. Error bars show the standard errors of the means across observers.

creased less sharply as a function of increased density for heterogeneous displays than for homogeneous displays.

Figure 5 shows the probability of reporting a given disk's color as a function of position of that disk relative to the target for the 0-step (Figure 5A) and multiple-step (Figure 5B) conditions. As in Experiment 1, there were very few errors in the 0 -step condition. Where errors occurred in the multiple-step condition, they were mainly reports of the color of a disk one disk away from the target.

\section{GENERAL DISCUSSION}

Using an attentional walk task, we found that shifting attention within heterogeneous arrays of items was more effective than shifting attention within homogeneous arrays of items. Most notably, arrays of densities for which the homogeneous condition was near chance nonetheless supported performance well above chance in the heterogeneous condition.

Before considering the implications of these findings, we will address the potential concern that the advantage observed for heterogeneous displays over homogeneous displays could be unique to the attentional walk task. In particular, the heterogeneous condition may have afforded improved guessing strategies for the attentional walk task that were unavailable or were less easily used in the homogeneous condition. This possibility seems unlikely for two different reasons. First, most of the errors were one-away errors (see Figures 2 and 4). This suggests that observers were not guessing very often, but instead were basing their responses on the identity of a particular (sometimes wrong) disk. If they were not guessing very often, then an opportunity for improved guessing strategies would have had little effect. Second and more generally, there was no heterogeneous advantage in the lowest density conditions, even when performance was below ceiling. If there were a general strategic advantage for the heterogeneous displays, then it should have appeared in these conditions as well. Based on these observations, we suggest that the advantage for heterogeneous displays over homogeneous displays reflects a greater ability to individuate items in the heterogeneous displays than in the homogeneous displays.

\section{Significance of the Influence of a Nonspatial Attribute on Spatial Selection}

The main implication of the finding that observers performed better on attentional walks in heterogeneous displays than in homogeneous displays is that the limitations of spatially shifting attention can be modulated by nonspatial features of stimuli (color in this case). Literature produced prior to this study has included empirical indications that nonspatial features could influence spatial selection and therefore could influence spatial shifting of attention. For example, interference on target identification from flankers in the response time task of C. W. Eriksen and colleagues (B. A. Eriksen \& C. W. Eriksen, 1974; C. W. Eriksen \& Hoffman, 1973) was reduced when the flankers were given features distinct from those of the target (Baylis \& Driver, 1992). Moreover, visual crowding is modulated by the similarity between the flankers and the target (i.e., there is more crowding when the flankers are more similar to the targets; Kooi, Toet, Tripathy, \& Levi, 1994; Nazir, 1992). To the extent that visual crowding is related to the spatial limitations of selective processing, these findings suggest that nonspatial attributes can influence spatial selection. What distinguishes the effect of nonspatial features in the present study from these previous observations is that performance in the attentional walk task required that items be individuated but not identified. Identification, unlike individuation, requires that the appropriate features be associated with the appropriate objects. In contrast, shifting attention from one item to another does not, in principle, require the reliable integration of features. Nonetheless, the present results suggest that the process of integration can facilitate the individuation of items for purposes of shifting attention to them.

In addition to empirical indications, intuition suggests that the addition of differentiating feature information should facilitate individuation. When discussing the attentional walk task in the broader context of findings reported in literature on visual crowding, Intriligator and Cavanagh (2001) wrote that "whenever adjacent items differ in some salient feature (e.g., different shapes, different sizes or colors) they will be easier to isolate" (p. 202). In order to accommodate such facilitation theoretically, however, the specific mechanisms by which the limits of spatial selection arise need to be considered, since, as noted in the introduction, the basic construct of attentional resolution does not predict facilitation due to distinctive features.

Intriligator and Cavanagh (2001) suggested a general mechanism that, when considered within the context of specific models of visual processing, could accommodate the present findings. In particular, they suggested that the limits of attentional resolution may be determined by characteristics of neurons that have relatively large receptive fields (e.g., those in parietal cortex relative to 

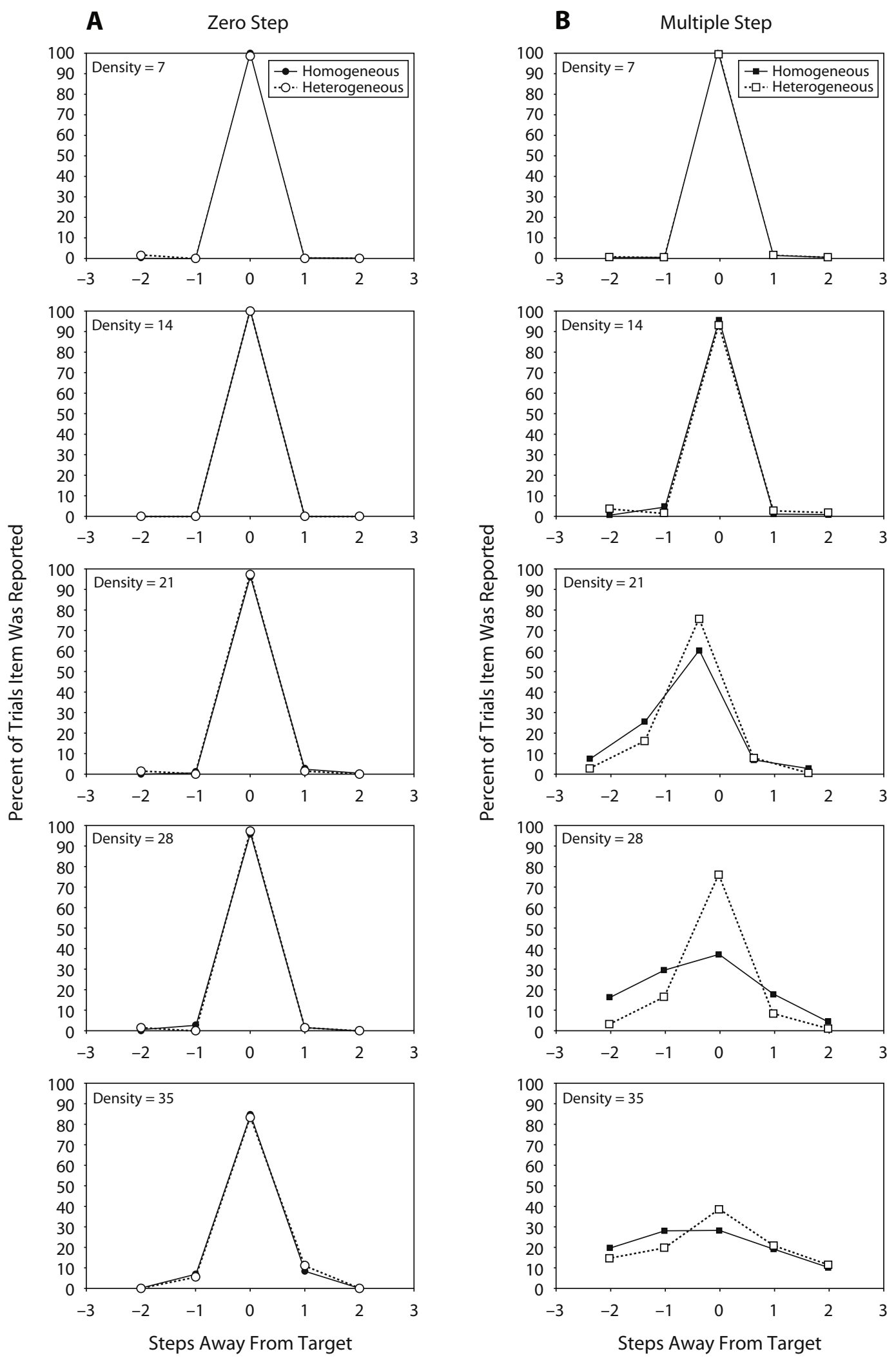

Figure 5. Probability of reporting the color of a given disk as a function of that disk's distance from the target disk for Experiment 2. Filled symbols represent homogeneous displays and open symbols represent heterogeneous displays. (A) Data for five different density levels in the 0-step conditions. (B) Data for five different density levels in the multiple-step conditions. 
those in area V1). If multiple stimuli fall within receptive fields of different neurons, the stimuli can be represented distinctly from each other by the two neurons. However, as noted many times in the past, if multiple stimuli fall within the receptive field of a single neuron, then they cannot be distinctly represented by that neuron without additional modulation (for a review see, e.g., Luck \& Beach, 1998). Attention has been implicated in several models as a means for resolving representational ambiguity. For example, one well-known model, the biased competition model (Desimone, 1998; Desimone \& Duncan, 1995), takes as a starting assumption that when multiple stimuli appear at the same time, they compete for neural representation. If there is relatively little overlap among the neural substrates that two stimuli activate (e.g., if one stimulus is a white square presented in the left visual field and the other is a tone played close to the right ear), then the competition will be of little consequence. The more overlap there is, however, the more competition there is. According to the model, attending to a particular stimulus can bias the competition for neural representation in favor of the attended stimulus. At a single-unit level, for example, imagine that two stimuli fall within the same receptive field of a given neuron in V4. That neuron receives input from other neurons that respond to only one of the stimuli. The synapses between those neurons and this specific V4 neuron comprise a site at which the competition for representation between the two stimuli can be biased; e.g., the synaptic weights for the neurons that responded to the attended stimulus can be increased relative to those that responded to the unattended stimulus (see Reynolds, Chelazzi, \& Desimone, 1999, for a limited implementation of this model).

To date, the biased competition model has focused primarily on situations in which selection is based on location. Conceptually extending this idea, it follows that more distinct stimuli will have more distinct neural representations at initial stages, and therefore the biasing process can be more effective. To be clear, consider an extreme theoretical situation. If two stimuli activated identical networks of neurons prior to synapsing with a target neuron for which the stimuli were competing, then biasing the input from one stimulus over the other would change nothing, because the same network of inputs would be influenced by the biasing process. In contrast, if the two stimuli activated at least partially different neural networks, then biasing the inputs from one stimulus over the other could influence which stimulus ended up driving the target neuron. Applying these ideas to the present results suggests that adjacent disks in heterogeneous displays activated neural networks that were more distinct from each other than those activated by adjacent disks in homogeneous displays. Therefore, the heterogeneous displays afforded greater opportunity for attending to one disk to be effective than did the homogeneous displays.

There is substantial empirical support for the general idea of biased competition. At the single cell level, changes in neural selectivity that are well characterized by a biased competition model have been found in V2 (Reynolds et al., 1999), V4 (see, e.g., Chelazzi, Miller,
Duncan, \& Desimone, 2001; Luck, Chelazzi, Hillyard, \& Desimone, 1997; Reynolds et al., 1999), frontal eye fields (see, e.g., Schall \& Hanes, 1993; see Thompson, Bichot, \& Schall, 2001, for a review), inferotemporal cortex (Chelazzi, Duncan, Miller, \& Desimone, 1998), and superior colliculus (see, e.g., Goldberg \& Wurtz, 1972). In addition, evidence from neural imaging studies shows changes in responsiveness in human cortical areas that are consistent with biased competition among stimuli (see Kastner \& Ungerleider, 2001, for a review). Finally, the basic ideas underlying biased competition, more broadly construed, exist in a variety of models of visual attention (Bundesen, 1990; Bundesen, Habekost, \& Kyllingsbæk, 2005), visual search (Wolfe, 1994), and saccade target selection (Findlay \& Walker, 1999; Schall, 2004). Although these models vary in terms of their level of analysis, degree of implementation, and original purpose, there is a convergence of ideas in terms of competition for representation that attention serves to resolve.

In addition to these empirical findings regarding competition, the notion of competition also exists in a number of models, including an implemented one developed by Tsotsos and colleagues (Tstotsos et al., 1995). Their model goes beyond the biased competition model of Desimone and colleagues to accommodate competition on the basis of representations of features in addition to location. The current data fit well with this extended notion of competition. In particular, the current data suggest that failures in the attentional walk task may reflect an inability of an attentional biasing process to resolve a competition between multiple stimuli for selective representation. Providing distinguishing feature information can facilitate this biasing process. Conditions in which the competition cannot be resolved result in a nonstable state of selection. This instability may be what is experienced at a phenomenological level when one is trying, but noticeably failing, to attend to one particular item in a dense array (see Intriligator \& Cavanagh, 2001, for a demonstration). This instability of selection may also be what is experienced at a phenomenological level when one perceives a jumble of letters in a visual crowding display (see Pelli et al., 2004, for a demonstration). Although the current results cannot speak directly to the relationship between attentional resolution and visual crowding, ongoing discussions of possible links can be found in Cavanagh (2001); Fine, Yurgenson, and Moore (2007); He et al. (1997); Pelli et al. (2004); and Strasburger (2005).

\section{AUTHOR NOTE}

This work was supported by NIH/NIMH Grant MH067793 to C.M.M. The authors are grateful to John Palmer and Toby Mordkoff for helpful discussions concerning this and related work. Thanks also to Scott Gustas for assistance in preparing the experimental code. Correspondence should be addressed to C. M. Moore, Department of Psychology, Pennsylvania State University, 614 Moore Building, University Park, PA 16802 (e-mail: cmm15@psu.edu).

\section{REFERENCES}

BaHCall, D. O., \& Kowler, E. (1999). Attentional interference at small spatial separations. Vision Research, 39, 71-86.

BaYlis, G. C., \& Driver, J. (1992). Visual parsing and response com- 
petition: The effect of grouping factors. Perception \& Psychophysics, 51, 145-162.

Bouma, H. (1970). Interaction effects in parafoveal letter recognition. Nature, 226, 177-178.

Brainard, D. H. (1997). The Psychophysics Toolbox. Spatial Vision, 10, 433-436.

Bundesen, C. (1990). A theory of visual attention. Psychological Review, 97, 523-547.

Bundesen, C., Habekost, T., \& Kyllingsbæk, S. (2005). A neural theory of visual attention: Bridging cognition and neurophysiology. Psychological Review, 112, 291-328.

Cavanagh, P. (2001). Seeing the forest but not the trees. Nature Neuroscience, 4, 673-674.

CAVE, K. R., \& Bichot, N. P. (1999). Visuospatial attention: Beyond a spotlight model. Psychonomic Bulletin \& Review, 6, 204-223.

Chelazzi, L., Duncan, J., Miller, E. K., Desimone, R., (1998). Responses of neurons in inferior temporal cortex during memory-guided visual search. Journal of Neurophysiology, 80, 2918-2940.

Chelazzi, L., Miller, E. K., Duncan, J., \& Desimone, R. (2001). Responses of neurons in macaque area V4 during memory-guided visual search. Cerebral Cortex, 11, 761-772.

Cutzu, F., \& Tsotsos, J. K. (2003). The selective tuning model of attention: Psychophysical evidence for a suppressive annulus around an attended item. Vision Research, 43, 205-219.

Desimone, R. (1998). Visual attention mediated by biased competition in extrastriate visual cortex. Philosophical Transactions of the Royal Society of London: Series B, 353, 1245-1255.

Desimone, R. \& Duncan, J. (1995). Neural mechanisms of selective visual attention. Annual Review Neuroscience, 18, 193-222.

ERIKSEN, B. A., \& ERIKSEN, C. W. (1974). Effects of noise letters upon the identification of a target letter in a nonsearch task. Perception \& Psychophysics, 16, 143-149.

Eriksen, C. W., \& Hoffman, J. E. (1973). Temporal and spatial characteristics of selective encoding from visual displays. Perception \& Psychophysics, 12, 201-204.

Eriksen, C. W., \& St. James, J. D. (1986). Visual attention within and around the field of focal attention: A zoom lens model. Perception \& Psychophysics, 40, 225-240.

ERIKSEN, C. W., \& YeH, Y.-Y. (1985). Allocation of attention in the visual field. Journal of Experimental Psychology: Human Perception \& Performance, 11, 583-597.

Fernandez-Duque, D., \& Johnson, M. L. (1999). Attention metaphors: How metaphors guide the cognitive psychology of attention. Cognitive Science, 23, 83-116.

FindLAY, J. M., \& WALKER, R. (1999). A model of saccade generation based on parallel processing and competitive inhibition. Behavioral \& Brain Sciences, 22, 661-674.

Fine, E. M., Yurgenson, S., \& Moore, C. M. (2007). Evidence of saccadic crowding: Nearby distractors decrease saccade accuracy. Manuscript submitted for publication.

GoldBerg, M. E., \& WURTZ, R. H. (1972). Activity of superior colliculus in behaving monkey: II. Effect of attention on neuronal responses. Journal of Neurophysiology, 35, 560-574.

He, S., Cavanagh, P., \& InTRILIGator, J. (1996). Attentional resolution and the locus of visual awareness. Nature, 383, 334-337.

He, S., Cavanagh, P., \& Intriligator, J. (1997). Attentional resolution. Trends in Cognitive Sciences, 1, 115-121.

Intriligator, J., \& CAVANAGH, P. (2001). The spatial resolution of visual attention. Cognitive Psychology, 43, 171-216.

Kastner, S., Ungerleider, \& L. G. (2001). The neural basis of biased competition in human visual cortex. Neuropsychologia, 39, 1263-1276.

Kooi, F. L., Toet, A., Tripathy, S. P., \& Levi, D. M. (1994). The effect of similarity and duration on spatial interaction in peripheral vision. Spatial Vision, 8, 255-279.

LaBerge, D., \& Brown, V. (1989). Theory of attentional operations in shape identification. Psychological Review, 96, 101-124.
LuCK, S. J., \& BEACH, N. J. (1998). Visual attention and the binding problem: A neurological perspective. In R. D. Wright (Ed.), Visual attention (pp. 455-478). New York: Oxford University Press.

Luck, S. J., Chelazzi, L., Hillyard, S. A., \& Desimone, R. (1997). Neural mechanisms of spatial selective attention in areas V1, V2, and V4 of macaque visual cortex. Journal of Neurophysiology, 77, $24-42$.

Moore, C. M., Lanagan-Leitzel, \& Fine, E. M. (2007) Attentional resolution as measured in the attentional walk task reflects limitations of control not spatial extent. Manuscript submitted for publication.

Mounts, J. R. W. (2000). Evidence for suppressive mechanisms in attentional selection: Feature singletons produce inhibitory surrounds. Perception \& Psychophysics, 62, 969-983.

NAZIR, T. A. (1992). Effects of lateral masking and spatial precueing on gap-resolution in central and peripheral vision. Vision Research, 32, 771-777.

Parkes, L., Lund, J., Angelucci, A., Solomon, J. A., \& Morgan, M. (2001). Compulsory averaging of crowded orientation signals in human vision. Nature Neuroscience, 4, 739-744.

PeLLI,D. G. (1997). The VideoToolbox software for visual psychophysics: Transforming numbers into movies. Spatial Vision, 10, 437-442.

Pelli, D. G., Palomares, M., \& MajaJ, N. J. (2004). Crowding is unlike ordinary masking: Distinguishing feature integration from detection. Journal of Vision, 4, 1136-1169.

Posner, M. I. (1980). Orienting of attention. Quarterly Journal of Experimental Psychology, 32, 3-25.

Posner, M. I., SNyder, C. R., \& Davidson, B. J. (1980). Attention and the detection of signals. Journal of Experimental Psychology: General, 109, 160-174.

Reynolds, J. H., Chelazzi, L., Desimone, R. (1999). Competitive mechanisms subserve attention in macaque areas V2 and V4. Journal of Neuroscience, 19, 1736-1753.

SCHALL, J. D. (2004). Selection of targets for saccadic eye movements. In L. M. Chalupa \& J. S. Werner (Eds.), The visual neurosciences (pp. 1369-1390). Cambridge, MA: MIT Press.

Schall, J. D., \& Hanes, D. P. (1993). Neural basis of saccade target selection in frontal eye field during visual search. Nature, 366, 467-469.

Steinman, B. A., Steinman, S. B., \& Lehmkuhle, S. (1995). Visual attention mechanisms show a center-surround organization. Vision Research, 35, 1859-1869.

Strasburger, H. (2005). Unfocussed spatial attention underlies the crowding effect in indirect form vision. Journal of Vision, $\mathbf{5}$ 1024-1037.

Thompson, K. G., Bichot, N. P., \& Schall, J. D. (2001). From attention to action in frontal cortex. In J. Braun, C. Koch, \& J. L. Davis (Eds.), Visual attention and cortical circuits (pp. 137-157). Cambridge, MA: MIT Press.

Tsotsos, J. K., Culhane, S. M., WaI, W. Y. K., LaI, Y. H., Davis, N., \& Nuflo, F. (1995). Modeling visual attention via selective tuning. Artificial Intelligence, 78, 507-545.

Wolfe, J. M. (1994). Guided Search 2.0: A revised model of visual search. Psychonomic Bulletin \& Review, 1, 202-238.

\section{NOTE}

1. Because the different colors were not equiluminant, differences in color were confounded with differences in luminance. Both hue and luminance are nonspatial features in the sense that we are using the term. For simplicity, we use the term color to refer to these nonspatial features.

(Manuscript received July 26, 2005; revision accepted for publication July 14, 2006.) 